\title{
Renaissance der Markteingriffe?
}

\author{
Henning Klodt
}

Online publiziert: 17 . November 2020

(C) List-Gesellschaft e.V. 2020

Der Wirtschaftspolitische Ausschuss des Vereins für Socialpolitik hatte sich für seine Jahrestagung 2020 ein Generalthema gewählt, das seit Covid-19 eher noch an Bedeutung gewonnen hat - die staatlichen Markteingriffe. Bei der Konzeption der Tagung war das Thema noch mit einem Fragezeichen versehen worden, doch in den einzelnen Beiträgen wurde deutlich, dass es in den im Einzelnen diskutierten Bereichen zumeist entfallen kann. Sowohl in den Vorträgen als auch in den Diskussionen dazu wurde allerdings deutlich, dass per se kaum beurteilt werden kann, ob mehr Staatseingriffe gesellschaftlich vorteilhaft oder nachteilhaft sind. Oftmals steckt der Teufel im Detail, d.h. es kommt weniger auf das $o b$ und mehr auf das wie an.

Eine Kernfrage dazu, die Ökonomen seit Jahrhunderten umtreibt, stellen Motivation und Rechtfertigung staatlicher Eingriffe in die Einkommensverteilung dar. Hagen Krämer verfolgt die Spuren dieser Diskussion sogar bis in die Antike zurück. Er plädiert dafür, das übliche Schema von Primär- und Sekundärverteilung zu erweitern um Aspekte, die die Entstehung der Primäreinkommen selbst beeinflussen. Dazu unterscheidet er zwischen pre-production (Ressourcenausstattung), production (u. a. technischer Wandel) und post-production (Umverteilung im engeren Sinne). Er plädiert für einen umfassenden Ansatz in der Verteilungspolitik, der insbesondere die Produktionssphäre nicht ausklammern sollte, da auf dieser Ebene oftmals irreversible Verteilungseffekte entstehen. Zugleich weist er aber darauf hin, dass ein allgemeiner Konsens darüber, wie eine faire Verteilung konkret aussehen sollte, nie erreichbar sein wird.

In diesem Heft wird berichtet über die Jahrestagung 2020 des Wirtschaftspolitischen Ausschusses des Vereins für Socialpolitik, die vom 3. bis 5. März 2020 an der Freien Universität Berlin stattfand. Ein besonderer Dank gilt Christian von Hirschhausen, der für die organisatorische Vorbereitung und Durchführung der Tagung verantwortlich war.

H. Klodt $(\bowtie)$

Kiel, Deutschland

E-Mail: henning.klodt@freenet.de 
Die Frage, welche Art von Markteingriffen adäquat ist, stellt sich zurzeit gerade im Bereich der Wettbewerbspolitik auf neue Weise. Die digitale Wirtschaft wird zunehmend von internationalen Monopolen dominiert, die den Marktwettbewerb durch Praktiken gefährden, die der herkömmlichen Wettbewerbspolitik nur schwer zugänglich sind. Oliver Budzinski ging in seinem Vortrag insbesondere der Frage nach, wie die im Rahmen der 10. GWB-Novelle vorgesehene Ausweitung der Missbrauchsaufsicht in der Digitalwirtschaft aus Sicht der modernen Wirtschaftstheorie zu beurteilen ist. Er plädiert dafür, anstatt der Ex-post-Verhaltenskontrolle der Missbrauchsaufsicht besser die Ex-ante-Strukturkontrolle zu schärfen, indem bei der kartellrechtlichen Beurteilung von Zusammenschlussvorhaben im Bereich der Digitalwirtschaft stärker auf marktübergreifende Aspekte geschaut wird.

Markteingriffe scheinen auch im Bereich des internationalen Handels auf dem Vormarsch zu sein. Jürgen Jerger zeigt anhand eines einfachen spieltheoretischen Ansatzes, dass Politiker oftmals dazu tendieren, ein mögliches Gefangenendilemma nicht durch multilaterale Handelsliberalisierungen zu lösen, wenn sie oder die Öffentlichkeit die Wahrnehmung haben, in ihrem Land gäbe es überdurchschnittlich viele Globalisierungsverlierer. Dann könne sogar die Gefahr einer weiteren Eskalation von Protektionsmaßnahmen drohen. Um die Handelspolitik vom Protektionsgleichgewicht hin zu einem Freihandelsgleichgewicht $\mathrm{zu}$ bewegen, sollten Ökonomen die Vorteile des Freihandels stärker in die öffentliche Diskussion einbringen.

Ein Bereich, der im Inland derzeit im Fokus der öffentlichen Debatte steht, ist der Breitbandausbau. Die Tendenzen zur Digitalisierung der Wirtschaft und des gesellschaftlichen Lebens sind schon länger angelegt, haben aber durch Covid-19 einen zusätzlichen Schub erfahren. Gordon Klein stellt in seinem Beitrag den aktuellen Stand des Breitbandausbaus in Deutschland dar und evaluiert am Beispiel des Landes Nordrhein-Westfalen die wesentlichen Instrumente der staatlichen Förderung in diesem Bereich. Grundsätzlich hält er diese Förderung für durchaus berechtigt, da im Breitbandbereich vielfältige positive Netzwerk-Externalitäten auftreten. Er plädiert allerdings dafür, mögliche Ausbauhemmnisse detaillierter als bisher zu erfassen, um die Förderinstrumente zielgenauer einsetzen zu können.

Eher zu wenige Markteingriffe gibt es dagegen im Straßenverkehr - zumindest dort, wo es um die Bepreisung knappen Verkehrsraums geht. Diese Ansicht vertritt Gernot Sieg in seinem Beitrag, der als Plädoyer für den verstärkten Einsatz von Maut-Systemen verstanden werden kann. Sieg unterscheidet zwischen Knappheiten auf Fernstraßen, die als Flaschenhals modelliert werden können, und Knappheiten in Städten, für die das Badewannenmodell adäquater ist. Von Ökonomen wird traditionell eine lastabhängige Preisdifferenzierung bei Mautgebühren empfohlen. Sieg weist allerdings darauf hin, dass die damit intendierte Verhaltensänderung der Verkehrsteilnehmer zunehmend auch über differenzierte Fahrzeitberechnungen in Navigationsapps erreicht wird. Damit könnte eine Regulierung der Verkehrsflüsse über den Preis redundant werden. Gleichwohl empfiehlt der Autor, einen einheitlichen europäischen Rechtsrahmen für Maut-Systeme zu schaffen, um die Handlungsoptionen der Verkehrspolitik auf regionaler und lokaler Ebene zu verbessern.

Jochen Michaelis und Benjamin Schwanebeck analysieren in ihrem Beitrag, wer die Gewinner und Verlierer der Einführung des Bestellerprinzips auf dem Mietwoh- 
nungsmarkt sind. Es könnte vermutet werden, dass es für die letztendliche Belastung der verschiedenen Marktparteien unerheblich sei, ob der Mieter oder der Vermieter die Maklerprovision zu zahlen hat. In einem sequentiellen spieltheoretischen Modell zeigen die Autoren jedoch, dass die Mieter eindeutig zu den Gewinnern zählen, da die Courtage, die sie zuvor meist allein zahlen mussten, heute nur noch unvollständig auf sie überwälzt wird. Aber auch die Vermieter gewinnen tendenziell, da die durchschnittlichen Courtagen sinken. Verlierer sind demnach allein die Makler. Hier wird es zu Marktaustritten kommen, so dass sich in der zweiten Runde höhere Suchkosten für Mieter und Vermieter ergeben. Bei plausiblen Parameterkonstellationen dürften die Grundaussagen des Modells dadurch allerdings nicht beeinträchtigt werden.

Mit zunehmender Alterung der Bevölkerung gewinnt die Raumstruktur des Angebots von Pflegedienstleistungen zunehmend an Bedeutung. Aufgrund hoher Immobilienpreise in den Ballungszentren ist dort kaum noch ein rentables Angebot von Pflegedienstleistungen möglich. Zugleich ist aber eine verstärkte Wanderungsbewegung Älterer in die Zentren zu beobachten. Dort ist also der größte Nachfrageanstieg nach Pflegedienstleistungen zu erwarten. Tobias Just und Franziska Plößl analysieren in ihrem Beitrag diese Entwicklungen und empfehlen, auf diese regionalen Ungleichgewichte mit einer verbesserten Bereitstellung von Baugrundstücken für Pflegeeinrichtungen in Städten und eventuell auch mit regional differenzierten Pflegesätzen zu reagieren.

Den Abschluss bildet ein Thesenpapier von Thieß Petersen, in dem es um fünf zentrale Herausforderungen für die künftige Wirtschaftspolitik geht. Als erstes nennt er den zunehmenden Druck auf die internationale Handelsordnung, die möglicherweise gar in einen Prozess der De-Globalisierung münden könnte. Die zweite Herausforderung erkennt er darin, dass der gesellschaftliche Konsens über das Wachstum von Wirtschaft und Wohlstand zunehmend erodiert. Die dritte Herausforderung liegt seines Erachtens darin, dass Verteilungskonflikte nicht nur schärfer, sondern auch komplexer werden. Dadurch könnten sich Partikularinteressen zum Nachteil der Gesamtwohlfahrt leichter durchsetzen. Viertens würden die politischen Gestaltungsmöglichkeiten von Nationalstaaten immer eingeschränkter, und fünftens gerieten Marktwirtschaften unter zunehmenden Druck aus Schwellenländern, die sich nicht an marktwirtschaftliche Regeln halten.

Insgesamt bot die Jahrestagung 2020 des wirtschaftspolitischen Ausschusses einen bunten Strauß an Politikanalysen aus den verschiedensten Bereichen, die allerdings durch eine gemeinsame Klammer zusammengehalten wurden: Hinter allen Analysen stand die Leitfrage, welche Rolle staatlichen Markteingriffen im Rahmen einer marktwirtschaftlichen Ordnung zukommt und wie ihre Effektivität und Effizienz mithilfe fundierter ökonomischer Analysen verbessert werden kann.

Henning Klodt Direktor am Institut für Weltwirtschaft a. D. 